ARTICLE

Received 28 May 2015 | Accepted 27 Oct 2015 | Published 1 Dec $2015 \quad$ DOl: 10.1038/ncomms10032 OPEN

\title{
High mobility emissive organic semiconductor
}

Jie Liu ${ }^{1,2, \star}$, Hantang Zhang ${ }^{1,2, \star}$, Huanli Dong ${ }^{1,2, \star}$, Lingqiang Meng ${ }^{3}$, Longfeng Jiang ${ }^{4}$, Lang Jiang ${ }^{5}$, Ying Wang ${ }^{3}$, Junsheng $\mathrm{Yu}^{4}$, Yanming Sun ${ }^{2}$, Wenping $\mathrm{Hu}^{1,2,6} \&$ Alan J. Heeger ${ }^{2}$

The integration of high charge carrier mobility and high luminescence in an organic semiconductor is challenging. However, there is need of such materials for organic light-emitting transistors and organic electrically pumped lasers. Here we show a novel organic semiconductor, 2,6-diphenylanthracene (DPA), which exhibits not only high emission with single crystal absolute florescence quantum yield of $41.2 \%$ but also high charge carrier mobility with single crystal mobility of $34 \mathrm{~cm}^{2} \mathrm{~V}^{-1} \mathrm{~s}^{-1}$. Organic light-emitting diodes (OLEDs) based on DPA give pure blue emission with brightness up to $6,627 \mathrm{cdm}^{-2}$ and turn-on voltage of $2.8 \mathrm{~V}$. 2,6-Diphenylanthracene OLED arrays are successfully driven by DPA field-effect transistor arrays, demonstrating that DPA is a high mobility emissive organic semiconductor with potential in organic optoelectronics.

\footnotetext{
${ }^{1}$ Beijing National Laboratory for Molecular Sciences, Key Laboratory of Organic Solids, Institute of Chemistry, Chinese Academy of Sciences, Beijing 100190, China. ${ }^{2}$ Heeger Beijing Research and Development Center, School of Chemistry and Environment, Beihang University, Beijing 100191, China. ${ }^{3}$ Key Laboratory of Photochemical Conversion and Optoelectronic Materials, Technical Institute of Physics and Chemistry, Chinese Academy of Sciences, Beijing 100190, China. ${ }^{4}$ School of Optoelectronic Information, University of Electronic Science and Technology of China, Chengdu 610054, China. ${ }^{5}$ Cavendish Laboratory, University of Cambridge, JJ Thomson Avenue, Cambridge CB3 OHE, UK. ${ }^{6}$ Department of Chemistry, School of Science, Tianjin University \& Collaborative Innovation Center of Chemical Science and Engineering (Tianjin), Tianjin 300072, China. ${ }^{\star}$ These authors contributed equally to this work. Correspondence and requests for materials should be addressed to H.D. (email: dhl522@iccas.ac.cn) or to W.H. (email: huwp@iccas.ac.cn) or to A.J.H.

(email: ajhe1@physics.ucsb.edu).
} 
T he achievement of organic semiconductors with both high mobility and strong fluorescence emission remains a challenge. High mobility requires molecules which pack densely and periodically, while serious fluorescence quenching typically occurs when fluorescent materials begin to aggregate (aggregation-induced quenching $(\mathrm{AIQ}))^{1-3}$. Indeed, classical materials with strong fluorescent emission always exhibit low mobility $^{4-7}$, for example, tris(8-hydroxyquinoline) aluminium $(\mathrm{ALQ})^{6}$ and phenylenevinylene-based polymers ${ }^{7}$ with mobility only $10^{-6}-10^{-5} \mathrm{~cm}^{2} \mathrm{~V}^{-1} \mathrm{~s}^{-1}$, and benchmark organic semiconductors with high mobility demonstrate very weak emission $^{8-11}$, for example, rubrene ${ }^{10}$ exhibits a quantum yield $<1 \%$ in crystalline state and pentacene ${ }^{11}$ shows very weak fluorescence in the solid state. However, organic semiconductors with high mobility and strong fluorescence are necessary for the achievement of high efficiency organic light-emitting transistors (OLETs) ${ }^{12-14}$ and electrically pumped organic lasers ${ }^{95,16}$.

Here, we demonstrate a high mobility organic semiconductor (2,6-diphenylanthracene, DPA) with strong emission: for single crystals, mobilities as high as $34 \mathrm{~cm}^{2} \mathrm{~V}^{-1} \mathrm{~s}^{-1}$ and photoluminescence quantum yield (PLQY) of $41.2 \%$ are obtained. OLEDs fabricated from this material give pure blue emission with brightness up to $6,627 \mathrm{~cd} \mathrm{~m}^{-2}$ with a turn-on voltage at $2.8 \mathrm{~V}$. Moreover, organic light-emitting diode (OLED) arrays of this compound can be driven by organic field-effect transistors (OFETs) fabricated with DPA. For the first time, we see the possibility of integration of optoelectronic devices based on the same organic semiconductor.

\section{Results}

The design synthesis and characterization of DPA. In the absence of significant intermolecular interactions (for example, in dilute solutions or when conjugated compounds are incorporated at very dilute concentrations in inert matrices), the radiative decay of these excitations are dominated by Kasha's rule, and the luminescence efficiency is relatively high ${ }^{1,2}$. However, strong intermolecular interactions, for example, $\pi-\pi$ co-facial stacking (H-aggregates), will cause the splitting of the lowest excited state (characterized by a non-emissive combination of the transition dipole moments of the individual molecule) resulting in optically forbidden transition to the ground state (AIQ). Thus, the luminescence is dramatically quenched (Supplementary Fig. 1) ${ }^{17}$. Therefore, the challenge for high mobility emissive organic semiconductors is how to balance intermolecular packing (for efficient charge transport) and non-radiative energy transfer (for efficient emission).

A novel compound, DPA (Fig. 1a), was designed and synthesized facilely with yield over $85 \%$ (Supplementary Fig. 2). The rational design takes into the consideration (i) the strong fluorescence of anthracene as the core, (ii) the phenyl groups at 2-, 6- positions of anthracene core are used to extend the molecular $\pi$ conjugation and tune the molecular packing. Indeed, the powder of DPA gives strong blue emission under UV light (Fig. 1b) with PLQY of $48.4 \%$ measured in a calibrated integrating sphere ${ }^{18}$. The high absolute quantum efficiency provides direct visual evidence of the strong emission of this compound.

To uncover the aggregation states of DPA solids, UV-vis and fluorescence measurements were conducted on DPA in different morphologies, including diluted solutions $(10 \mu \mathrm{M})$ in tetrahydrofuran (THF), nanoparticles dispersed in a THF: $\mathrm{H}_{2} \mathrm{O}=1: 9(\mathrm{v} / \mathrm{v})$ mixture, single crystals grown by physical vapour transport (PVT) technique ${ }^{19}$ and vacuum-deposited thin films on a quartz plate. The use of nanoparticles is beneficial for determination of aggregation state of chromophores ${ }^{20-22}$. Here we included UV-vis and PL spectra of DPA nanoparticles for comparison. As shown in Fig. 1c,d, the lowest $0 \rightarrow 0$ transition of DPA in diluted solution appears at $398 \mathrm{~nm}$ as a sharp absorption peak along with the $0 \rightarrow 1$ and $0 \rightarrow 2$ transitions at 377 and $360 \mathrm{~nm}$ (Fig. 1c, black line). The emission maximum of the DPA in solution (Fig. 1d, black line) appears at $408 \mathrm{~nm}$, yielding a Stokes shift of about $615.8 \mathrm{~cm}^{-1}$. In comparison with DPA solution, the absorption and emission spectra of DPA nanoparticles demonstrate an obvious bathochromic shift with the maximum absorption peaks at $433 \mathrm{~nm}$ and the maximum emission peaks at $441 \mathrm{~nm}$, respectively. The calculated Stokes shift for DPA nanoparticles in solution is $\sim 419.0 \mathrm{~cm}^{-1}$, that is, much a

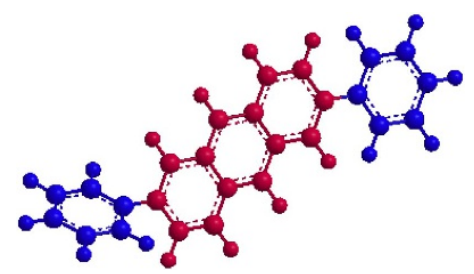

C

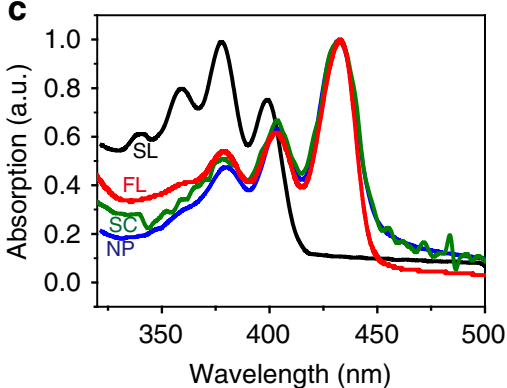

b

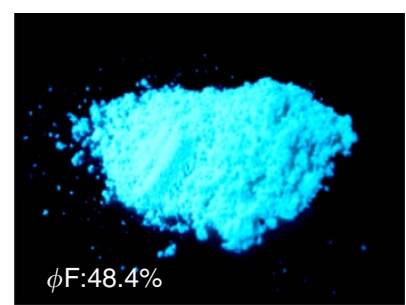

d

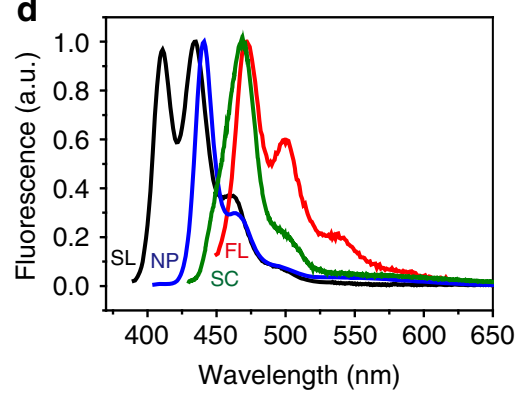

Figure 1 | Molecular structure of DPA and its corresponding absorption and emission features. (a) Molecular structure of DPA. (b) The powder of DPA gives strong blue emission under UV light with absolute fluorescent quantum yield up to $48.4 \%$. (c,d) UV-vis and fluorescent spectra of DPA monomers in diluted solution ( $\mathrm{SL}$, black line), DPA nanoparticles in a THF: $\mathrm{H}_{2} \mathrm{O}=1: 9(\mathrm{v} / \mathrm{v})$ mixture (NP, blue line), single crystals grown by PVT technique (SC, green line) and its vacuum-deposited thin film (FL, red line). 
smaller than that of DPA in dilute solution $\left(615.8 \mathrm{~cm}^{-1}\right)$. As for single crystals and thin films, larger bathochromic shifts as compared with nanoaggregates are observed in the fluorescent spectra, and the maximum emission peaks are at 468 and $472 \mathrm{~nm}$, respectively. Note that the absorption and emission spectra exhibit distinct vibronic sidebands. The $0-0$ peaks are much stronger than the corresponding $0-1$ or $0-2$ transitions in solid states. The increased ratio of the $0-0$ vibronic peak intensities compared with the $0-1$ and $0-2$ peaks in solid states suggests the formation of J-aggregates of DPA ${ }^{23}$ which thereby minimize the fluorescence quenching in the solid state.

Strong blue emission (Fig. 2a) is observed from the crystals and high PLQY of 41.2\%. X-ray diffraction (XRD) patterns of DPA single crystals (Supplementary Fig. 3) show strong and sharp diffraction peaks, demonstrating the high quality of the crystals. Single crystal X-ray crystallographic results (crystal size: $\sim 0.20 \mathrm{~mm} \times 0.20 \mathrm{~mm} \times 0.10 \mathrm{~mm}$ ) demonstrate that DPA belongs to the $\mathrm{P} 2(1) / \mathrm{c}$ space group with crystal parameters of $a=17.973(8) \AA, \quad b=7.352(3) \AA, c=6.245(3) \AA$ and $\beta=90.646(9)^{\circ}$. Reflections of XRD results are indexed to be (h00) peaks according to the single crystal structure, which implies that the (h00) crystal plane is parallel to the surface of the substrate. Transmission electron microscopic (TEM) images of an individual hexagonal crystal and its corresponding selected area electron diffraction (SAED) pattern are shown in Fig. 2b,c. The SAED pattern is indexed with the lattice parameters obtained from bulk crystal: $a=17.973(8) \AA, b=7.352(3) \AA, c=6.245(3) \AA$. The crystal facets could be indexed directly according to the law of constancy of interfacial angles from the regular hexagonal single crystals. The sharp diffraction peaks, clear edges and well assigned diffraction spots indicate the high quality of the crystals.

Based on the structural information of the single crystals, the anthracene core of the DPA molecule is flat while the substituted phenyls are slightly out of the plane of conjugation with a torsion angle of $20.05^{\circ}$ (Supplementary Fig. 4a). In crystal, DPA molecules adopt a herringbone packing motif as J-aggregates (Fig. 2d and Supplementary Fig. 4b,c) which effectively minimize the quenching of the DPA fluorescence in the solid state $e^{17,24,25}$. More detailed structure analysis demonstrates that the transition dipole moment is identified to be nearly parallel to the long axis of DPA molecule, which results in the bright edge emission of crystals (Supplementary Fig. 5). In addition, multi- and strong intermolecular $\mathrm{C}-\mathrm{H}-\pi$ interactions (with intermolecular distance only 2.84-2.86 $\AA$ ) are observed between neighbouring DPA molecules (Supplementary Fig. 6). Assuming one molecule accepts a charge, it could transfer the accepted charge to its near two neighbours, and then to other neighbours quickly, finally forming a two-dimensional network of charge transport (Fig. 2d and Supplementary Fig. 7) 26,27 . These features are consistent with efficient charge transport and high mobility.

DPA single crystal field-effect transistors. To evaluate the charge transport properties of DPA, bottom-gate top-contact transistors based on individual DPA micrometre-sized crystals are fabricated with a 'ribbon-mask-technique ${ }^{\text {28,29 }}$ based on octadecyltri-
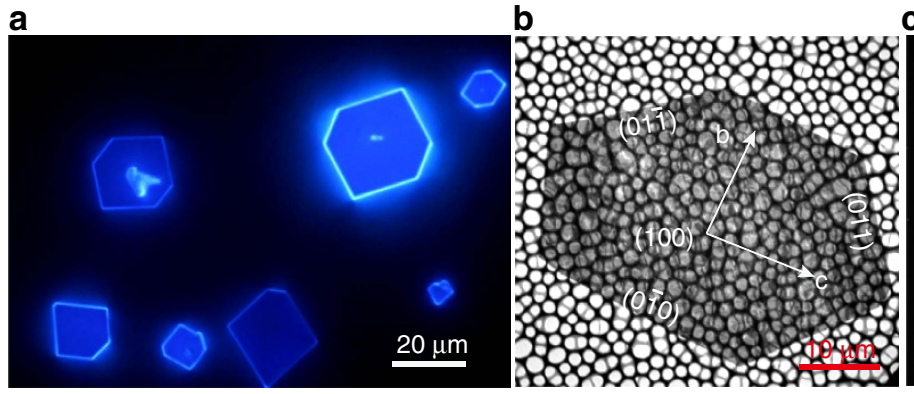

C

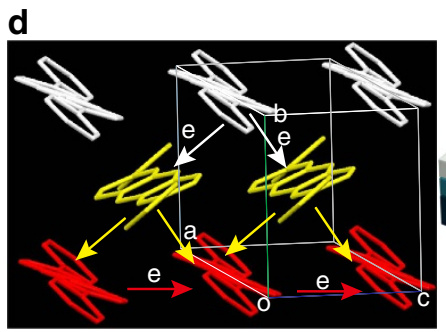

e

f
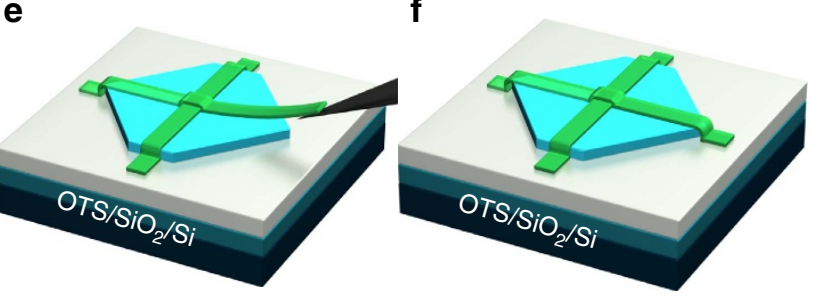

g

h

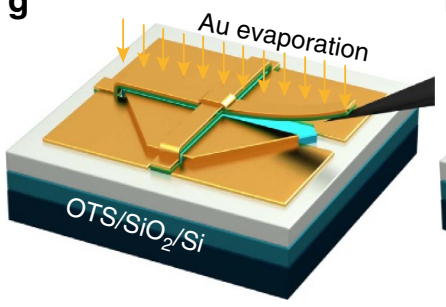

h

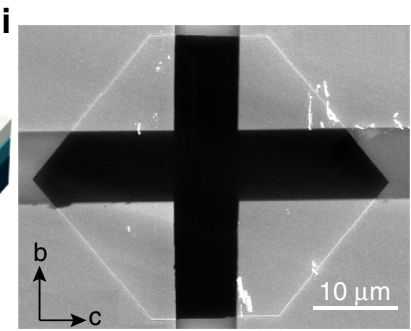

Figure 2 | DPA crystal features and device fabrication. (a) DPA crystals produced by PVT under UV light, the hexagonal crystals giving strong blue emissions. (b,c) Transmission electron microscopic image of individual DPA hexagonal crystal and its corresponding SAED pattern. (d) Charge transport along $b$ and $c$ axes in DPA crystal. (e-h) Fabrication of device based on individual micrometre-sized single crystal by 'organic ribbon mask' technique on n-doped Si substrate with $300 \mathrm{~nm} \mathrm{SiO}$, and OTS monolayer. (i) An SEM image of an example transistor fabricated by the 'organic ribbon mask' technique. 
chlorosilane (OTS)-treated $\mathrm{Si} / \mathrm{SiO}_{2}(300 \mathrm{~nm})$ substrates as shown in Fig. 2e-h. First, hexagonal single crystals of DPA are deposited on the substrate by PVT (Fig. 2e). Second, micrometre or submicrometre organic ribbons prepared in advance are used as the mask. An individual organic ribbon is peeled off and put directly on a pre-deposited DPA crystal, and then another organic ribbon is laminated over the previous one at $90^{\circ}$ (Fig. 2f). Third, $\mathrm{Au}$ is vacuum-deposited on the masked single crystal (Fig. $2 \mathrm{~g}$ ). Finally, the transistor with four crossed electrodes on an individual hexagonal crystal is obtained after the organic mask-ribbons are peeled off (Fig. 2h) (more details on device fabrication included in Methods). An example device based on an individual DPA crystal with four electrodes along $b$ and $c$ axes is shown in Fig. $2 \mathrm{i}$.

Transistors are measured on a Keithley 4200 SCS and Micromanipulator 6150 probe station with a high-resolution microscope (magnification at $\times 40-200$ ) (in air and at room temperature) and the mobility is extracted from the equation of $I_{\mathrm{DS}}=(W / 2 L) C_{\mathrm{i}} \mu\left(V_{\mathrm{G}}-V_{\mathrm{T}}\right)^{2}$. The typical transfer and output characteristics are shown in Fig. $3 \mathrm{a}-\mathrm{c}$ along the $b$ axis and Fig. 3d-f along the $c$ axis. Totally, 100 devices are examined, and over $95 \%$ devices exhibit mobility $>10 \mathrm{~cm}^{2} \mathrm{~V}^{-1} \mathrm{~s}^{-1}$ along $b$ axis (Fig. 3c) with the maximum mobility up to $34 \mathrm{~cm}^{2} \mathrm{~V}^{-1} \mathrm{~s}^{-1}$, while along $c$ axis only $75 \%$ devices demonstrate mobility over $10 \mathrm{~cm}^{2} \mathrm{~V}^{-1} \mathrm{~s}^{-1}$ with the maximum mobility up to $23 \mathrm{~cm}^{2} \mathrm{~V}^{-1} \mathrm{~s}^{-1}$ (Fig. 3f). We estimated $4.5 \mathrm{~cm}^{2} \mathrm{~V}^{-1} \mathrm{~s}^{-1}$ for the linear mobility based on the data from Fig. 3a using $16.5 \mu \mathrm{m}$ for the channel length and $3.3 \mu \mathrm{m}$ for the channel width (assuming that the gate induced charge was confined to either the first two or the first four monolayers) indicating a relatively large contact resistance.
The threshold voltage for the turn-on of the FETs is rather large, typically $\sim-20 \mathrm{~V}$, indicating a high density of traps at the dielectric interface. Note also that there is a mismatch between the highest occupied molecular orbital (HOMO) energy level of DPA $(5.6 \mathrm{eV})$ and the work function of gold $(5.2 \mathrm{eV})$, which probably results in a relatively large contact resistance responsible for the initial curvature in the output data for $V_{\mathrm{DS}}<-5 \mathrm{~V}$ as shown in Fig. 3. Hence, further device optimization can be expected. The mobility anisotropy along different crystal directions is in the range of 1.3-1.5 along $b$ and $c$ axes (Supplementary Fig. 8).

DPA organic light-emitting diodes. As an additional proof to confirm the high emission from this novel compound, OLEDs of DPA are fabricated as shown in Fig. 4a. A $40 \mathrm{~nm}$ N,N'-di-[(1-naphthyl)-N,N'-diphenyl]-1,1' -biphenyl)-4,4'- diamine (NPB) layer is inserted between indium tin oxide (ITO) and the emitting molecular layer (EML) of DPA films (30 or $40 \mathrm{~nm}$ ) for energy level alignment to facilitate hole-injection and transport ${ }^{30}$. Here, DPA films are vacuum-deposited polycrystalline films composed of small crystalline domains as evidenced by their AFM images (Supplementary Fig. 9a), which have the same molecular packing structure and orientation as that of single crystals identified by the XRD results (Supplementary Figs 3 and $9 b)$. For efficient electron-injection, LiF is adopted as the buffer layer. Moreover, considering the high mobility of DPA for efficient hole transport, another $30 \mathrm{~nm} \mathrm{1,3,5-tri(p-pyrid-3-yl-}$ phenyl)benzene (TMPYPB) is used as a hole-blocking and electron transport layer between $\mathrm{LiF}$ and DPA, because of the a

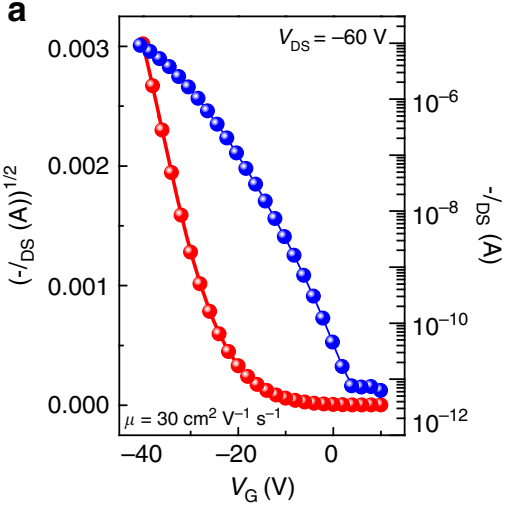

d

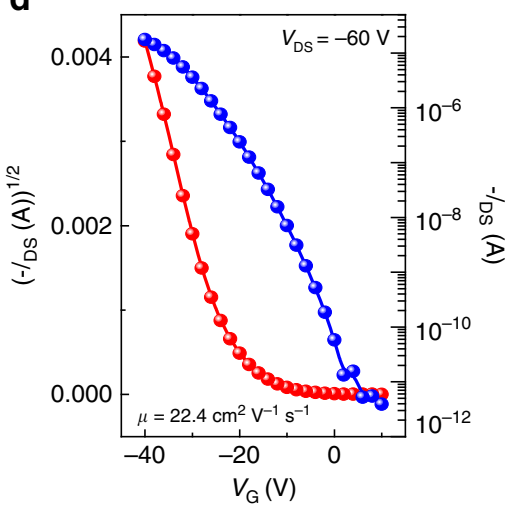

b

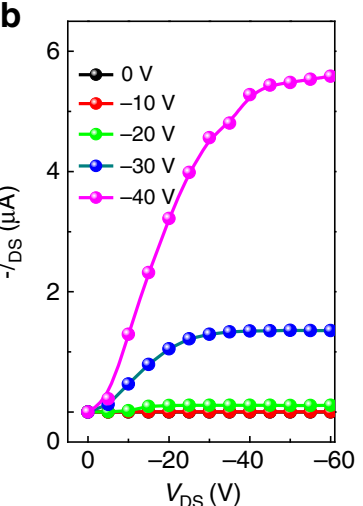

e

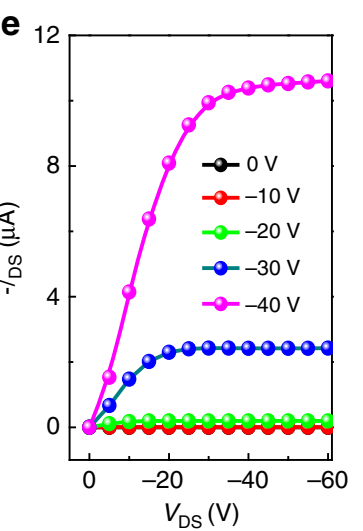

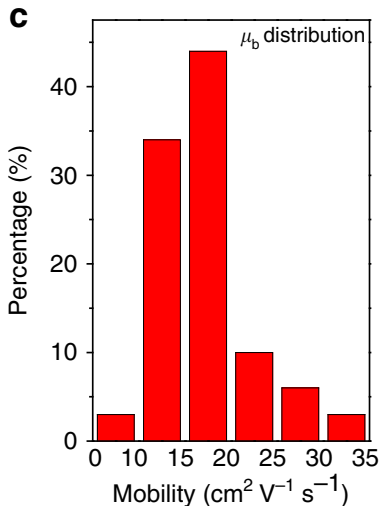

f

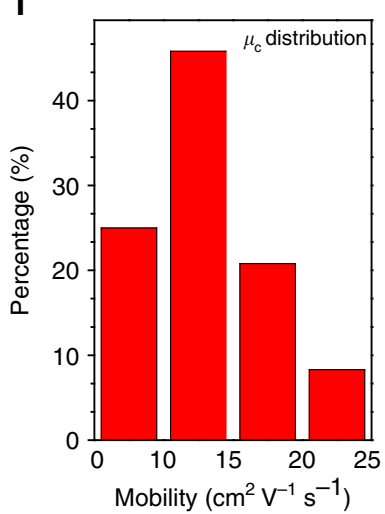

Figure 3 | Device performance of DPA single crystal transistors along $\boldsymbol{b}$ and $\mathbf{c}$ axes. $(\mathbf{a}, \mathbf{b})$ Typical transfer and output characteristics along $b$ axis, which give mobility of $30 \mathrm{~cm}^{2} \mathrm{~V}^{-1} \mathrm{~s}^{-1}$ with channel length of $16.5 \mu \mathrm{m}$ and width of $3.3 \mu \mathrm{m}$. (c) Mobility distribution along $b$ axis based on 56 devices. (d,e) Typical transfer and output characteristics along $c$ axis, which give mobility of $22.4 \mathrm{~cm}^{2} \mathrm{~V}^{-1} \mathrm{~s}^{-1}$ with channel length of $26.4 \mu \mathrm{m}$ and width of $12.1 \mu \mathrm{m}$. (f) Mobility distribution along $c$ axis based on 44 devices. 
a

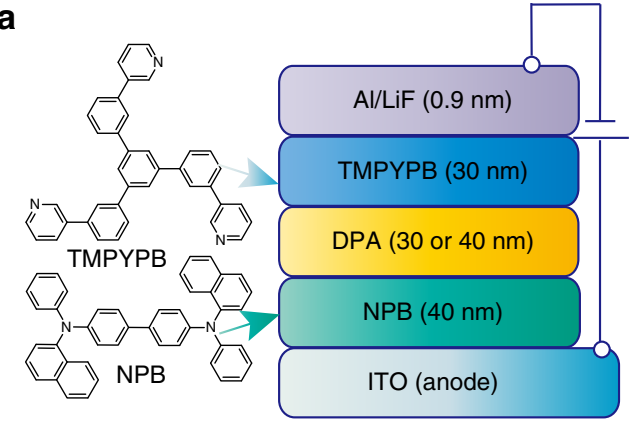

c

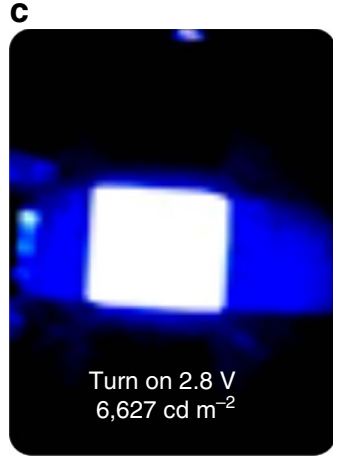

d

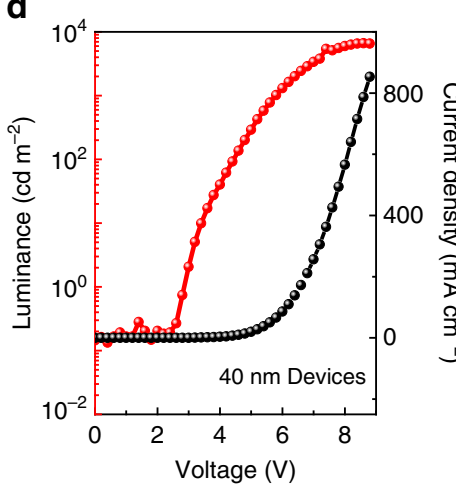

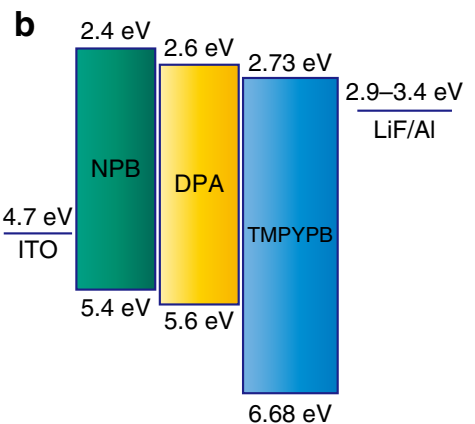

e



Figure 4 | OLEDs features of DPA. (a) OLEDs based on DPA with NPB, LiF and TMPYPB as buffer layers. (b) The energy-level diagram of OLEDs based on DPA. (c) Strong blue emission from OLEDs based on DPA $(2 \mathrm{~mm} \times 2 \mathrm{~mm})$. (d) Current density-voltage-luminance characteristics of the OLEDs.

(e) EL spectra operated at different voltages.

deep HOMO and low LUMO levels of TMPYPB. The energylevel diagram of the OLEDs is shown in Fig. 4b. The stepwise energy levels work efficiently for carrier injection and transport. Indeed, strong blue emission is observed from the DPA-based OLEDs as shown in Fig. 4c. A turn-on voltage of $2.8 \mathrm{~V}$ was achieved at the brightness of $1-10 \mathrm{~cd} \mathrm{~m}^{-2}$ and the maximum brightness was approaching $6,627 \mathrm{~cd} \mathrm{~m}^{-2}$ at $8.8 \mathrm{~V}$ when using $40 \mathrm{~nm}$ DPA as the active layer (Fig. 4d), which is higher than that of devices with $30 \mathrm{~nm}$ active layer (Supplementary Fig. 9c,d). Figure $4 \mathrm{e}$ shows the typical electroluminescence spectra (EL) of DPA-based OLEDs, which exhibits a sharp emission peak at $445 \mathrm{~nm}$. There is no obvious shift under different voltages with the full-width at half-maximum of $35 \mathrm{~nm}$. The OLED performance proves directly that DPA is an efficient blue emitter with low turn-on voltage and high brightness.

DPA integrated optoelectronic arrays. Integrated optoelectronic devices based on organic semiconductors is an essential goal for organic optoelectronic circuits ${ }^{31}$. One of the main obstacles is the lack of organic transistors with sufficiently high mobility and high $\mathrm{ON}-\mathrm{OFF}$ ratio to achieve reasonable switching speeds in logic circuits $^{32}$ and to drive OLEDs. The different complex processes for the fabrication of OFETs and OLEDs also present a challenge to device integration. Here, we demonstrate the integration of our optoelectronic devices based on the same organic semiconductor, DPA. As an example, we choose a simple pixel-like configuration in which OFETs fabricated from the same material supply the current to drive the OLED arrays. The schematic representation of the circuit is shown in Fig. 5a,b; and the OFET arrays driving the OLED arrays are shown in Fig. 5 c. Note that the devices in Fig. 5 are thin film devices with measured mobility of approximately $3-4 \mathrm{~cm}^{2} \mathrm{~V}^{-1} \mathrm{~s}^{-1}$. When an OFET is switched on, the current flowed from the source electrode to the OLED cathode, and hence, the OLED could be switched on and off by the OFET gate voltage $V_{\mathrm{G}}$ (for switching curves see Supplementary Fig. 10). A typical switched on OLED arrays are shown in Fig. 5d. By optimizing the device fabrication, at $V_{\mathrm{G}}=-70 \mathrm{~V}$, the OFET supplies a current density of $\sim 170 \mathrm{~mA} \mathrm{~cm}^{-2}$ to the OLED, resulting in a brightness at around $\sim 2,660 \mathrm{~cd} \mathrm{~m}^{-2}$ (Supplementary Fig. 11).

\section{Discussion}

For high performance OFETs, high mobility is essential, which requires molecules of organic semiconductors packing densely ${ }^{26,27}$. For example, $\mathrm{Hu}$ et al. found that molecules of $\alpha$-titanylphthalocyanine (TiOPc) possess ultra close $\pi$-stacking structure with N6-N6 distance of $3.145 \AA$ in convex pair and C9-C28 of 3.211 $\AA$ in concave pair, and hence thin film OFETs of $\alpha$-TiOPc exhibited the highest mobility of $10 \mathrm{~cm}^{2} \mathrm{~V}^{-1} \mathrm{~s}^{-1}$ and on/off ratio at $10^{7}$ (ref. 33). Bao and Locklin et al. found that the reduced molecular packing distance of TIPS-pentacene from 3.33 to $3.08 \AA$ by solution-shearing method could improve charge carrier mobility greatly from 0.8 to $4.6 \mathrm{~cm}^{2} \mathrm{~V}^{-1} \mathrm{~s}^{-1}$ (ref. 34). However, generally, densely packed organic semiconductors will bring strong intermolecular coupling, which results in serious fluorescence quenching, so that benchmark organic semiconductors with high mobility demonstrate very weak ${ }^{8-11}$ or even no emission ${ }^{33}$ in the solid state.

For OLEDs, organic semiconductor with strong emission is a pre-requirement in order to get high efficiency ${ }^{3-6}$. Moreover, it is necessary to limit the emission zone in the emissive layer and to have energy level alignment for well-balanced charge injection. Both are essential to provide efficient charge injection and carrier recombination for exciton formation and ultimately decay to the ground state with light emission. For the confinement of excitons in the active layer effectively, one needs charge carriers 


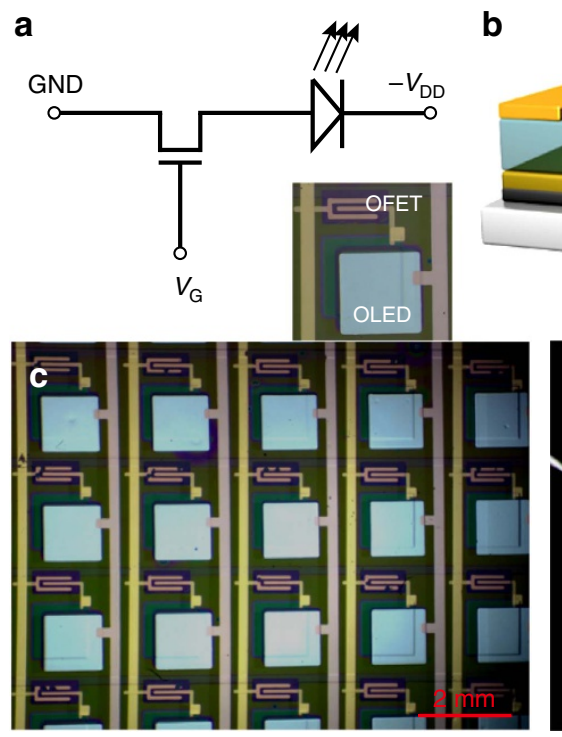

b
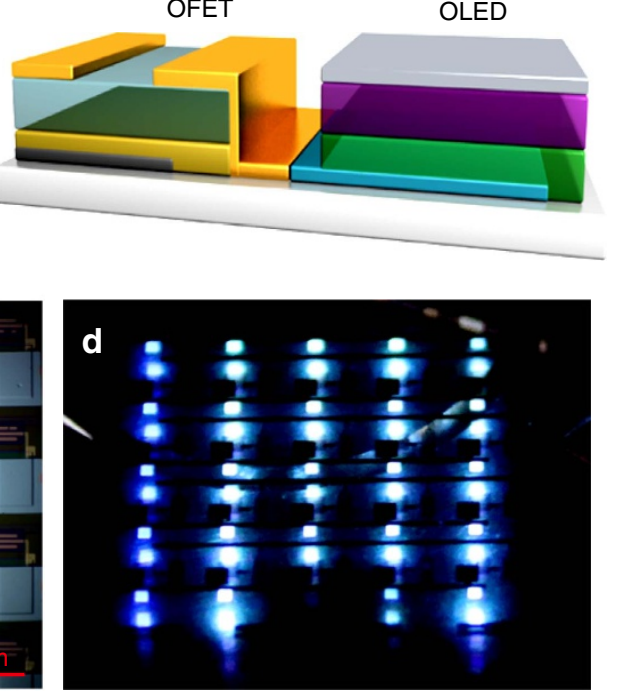

Figure 5 | Organic optoelectronic circuit of OFET driving OLED arrays based on DPA. (a,b) A configuration of OFETs driving OLED pixel, both OLED and OFET based on DPA. (c) Image of the OFET arrays driving OLED arrays. (d) Photo of the switched on DPA OLED arrays by DPA OFET arrays. GND, ground.

transporting not too fast in such thin active layer $(\sim 100 \mathrm{~nm})$, to provide enough time for exciton formation and decay, and hence low or moderate charge carrier mobility could meet the requirement. Hence, classical materials with strong fluorescent emission always exhibit low mobility ${ }^{4-7}$.

The OLET is a novel concept transistor that emits light ${ }^{12-14}$. The OLET integrates light emission from the OLED and electrical switching by the OFET. Consequently, except excellent luminescence properties, ideal field-effect properties (for example, mobility) are also critically for an OLET. Moreover, by an elaborate design, the OLET could achieve minimum excitonic losses, efficient light confinement and high current density during operation while maintaining the quantum efficiency, and hence is considered to be a promising route toward realizing electrically driven organic laser ${ }^{15,16}$. Therefore, organic semiconductors that can meet the criteria of high mobility and strong emission are needed.

According to exciton theory ${ }^{35,36}$, a fluorescent molecule is regarded as a dipole, and through the interaction of the two transition dipoles in a dimer, the excitonic state of the molecules splits into two levels. In an $\mathrm{H}$-aggregate, the parallel dipoles (as the arrows in Supplementary Fig. 1) repel each other leading to a higher energy state, while the anti-parallel dipoles of a J-aggregate attract each other and lower the energy of that state. The syntropic dipoles get the lower energy state, while the reversed dipoles increase the energy of that state ${ }^{37}$. It is well known that, fluorescence is generated from the transition from the lowest excited state to the ground state $\mathrm{s}^{37,38}$, for J-aggregates the lower exciton emission is allowed with a red-shifted fluorescence compared with the monomer, while the allowed transition in the $\mathrm{H}$-aggregate is to the upper level with a blue-shifted fluorescence, vibrational relaxation from the upper level to the lower one explains the lower luminescence efficiency of $\mathrm{H}$-aggregates ${ }^{20}$. Cornil et al. ${ }^{17,39}$ proved that by adjusting the long axes of adjacent molecules to tune the molecular packing or to shift molecular packing from $\mathrm{H}$-aggregation to J-aggregation works efficiently to prevent the quench of luminescence, because the dipole-dipole interactions in such molecular packing motifs are weakened. In our case, the non-planar DPA molecule (its anthracene core is flat while the substituted phenyls are slightly out of the conjugated plane with a torsion angle of $20.05^{\circ}$ ) indeed adopts J-aggregation, which works effectively to minimize the quenching of the fluorescence of DPA in solid state, so that the powder and crystal of DPA both give strong blue emission (Figs $1 \mathrm{~b}$ and 2a). Meanwhile, multi-/strong intermolecular $\mathrm{C}-\mathrm{H}-\pi$ interactions (with molecular distance only $2.84-2.86 \AA$ ) and compact molecular packing are observed in DPA crystals, that is, beneficial for efficient charge carrier transport. Assuming one molecule accepts a charge, it could transfer the accepted charge to its near two neighbours, and then to other neighbours quickly, finally forming a two-dimensional network of charge transport (Supplementary Fig. 7). Indeed, DPA exhibits not only bright blue emission (quantum efficiency of $41.2 \%$, electroluminescence $>6,600 \mathrm{cdm}^{-2}$ ), but also high hole mobility (up to $34 \mathrm{~cm}^{2} \mathrm{~V}^{-1} \mathrm{~s}^{-1}$ ). This proves that the goal to obtain high mobility emissive organic semiconductor is accessible. Moreover, DPA OLED arrays can be driven successfully by DPA OFET arrays. This demonstrates the successful integration of optoelectronic devices based on the same organic semiconductor, indicating the potential of DPA in organic optoelectronics, especially planar integration.

\section{Methods}

Material synthesis. DPA is synthesized through three facile steps with yields over 85\% (Supplementary Fig. 2). 2,6-dihydroxyanthracene-9,10-dione was purchased from Sigma Aldrich and was used as received. Triethylamine and dichloromethane (dried by molecular sieve) were used for the esterification reaction. Tetrakis (triphenylphosphine)palladium(0) and trifluoromethanesulfonic anhydride was purchased from Strem Chemicals and were used as received. Nuclear magnetic resonance (NMR) spectra were recorded using a Bruker ADVANCE 400 NMR Spectrometer and ${ }^{1} \mathrm{H}$ NMR spectra were referenced to $\mathrm{CDCl}_{3}$ (7.26 p.p.m.) Elemental analysis was carried out on Flash EA 1112 Elemental Analyser. AEI-MS50-MS spectrometer was used for molecular weight determination (more detailed synthesis information and characterizations see Supplementary Note 1).

UV-vis and PL spectra measurement. For Fig. 1b, the picture of DPA powder was taken under dark with a ultra-violet lamp shinning upon the compound with a wavelength of $365 \mathrm{~nm}$. The UV-vis absorption spectra were measured with Hitachi (model U-3010) UV-vis spectrophotometer in a $1 \mathrm{~cm}$ quartz cell for solution samples. Nanoparticles of DPA were prepared by injecting $1.8 \mathrm{ml} \mathrm{H}_{2} \mathrm{O}$ to a stirring system of $0.2 \mathrm{ml} 10^{-4} \mathrm{M}$ DPA solution in THF. Single crystals of DPA for UV-vis test were prepared by PVT technique with a sublimation temperature of $160^{\circ} \mathrm{C}$ and a deposition temperature of $50-60^{\circ} \mathrm{C}$ on quartz plate. And thin films of DPA samples for UV-vis test were fabricated by evaporation in high vacuum chamber on quartz plate under a pressure of $8 \times 10^{-4} \mathrm{~Pa}$, and the deposition rates were monitored using a quartz oscillating crystal and controlled at $0.1 \sim 0.2 \AA \mathrm{s}^{-1}$. And photoluminescence spectra were recorded on a Perkin-Elmer LS 55 spectro-

fluorometer, the excitation wavelength was $390 \mathrm{~nm}$ for solution sample, $395 \mathrm{~nm}$ for 
nanoparticles and $430 \mathrm{~nm}$ for single crystals and thin films. For solid state sample, LabSphere, FluoroMax-4, HORIBA JobinYvon, PLQY software package were used. The PLQY was measured using the integrating sphere in combination with a commercial fluorimeter and a repeatable value of $48.4 \%$ was obtained for DPA powder and $41.2 \%$ for single crystals (grown on a circular quartz plate with a diameter of $1 \mathrm{~cm}$ ). As for the thin films (PLQY, 19.5\%) deposited on quartz plate, the PLQY is smaller than the crystals, which might be caused by the defects and grain boundaries in thin film state. Emission spectra including the scattering region of excitation light were measured for both blank and test samples, and these spectra were corrected with instrumental factors to calculate the quantum yield. For solution, 9,10-diphenyl anthracene was used as reference and a relative quantum yield of $62.7 \%$ was determined. FL picture of the crystals was recorded by laser scanning confocal microscope (Olympus FV-1000-IX81) under mercury lamp with a charge-coupled device (CCD) camera.

Single crystal characterizations. For single crystal data analysis, large crystals of DPA are grown from saturated solution in chloroform. The crystal analysis was performed on Rigaku Saturn724 CCD at 113(2) K using MoK $\alpha$ with diffraction radiation wavelength of $0.71073 \AA$ and graphite as monochromator. For fluorescence property investigation and device fabrication, single crystals of DPA were grown by a physical vapour transport technique. Samples for laser scanning confocal microscope were grown on a $22 \mathrm{~mm} \times 22 \mathrm{~mm}$ cover slip, and FL pictures of the crystals was recorded by laser scanning confocal microscope (Olympus FV-1000-IX81) under mercury lamp with a CCD camera. Samples for XRD characterization was grown on $\mathrm{OTS} / \mathrm{SiO}_{2} / \mathrm{Si}$ substrates, considering the coverage of crystals on substrates, the substrate was turned four times with each time a different edge facing the sublimation source. X-ray diffraction measurement was performed in reflection mode at $40 \mathrm{kV}$ and $200 \mathrm{~mA}$ with $\mathrm{Cu}$ Ka radiation using a $2 \mathrm{~kW}$ Rigaku D/max-2500 X-ray diffractometer. Samples for TEM test were prepared by scratching crystals from $\mathrm{OTS} / \mathrm{SiO}_{2} / \mathrm{Si}$ and then transferred to copper grid with supporting carbon membrane. TEM and SAED measurements were carried out on a JEOL 2010 (Japan).

Fabrication and measurement of DPA single-crystal OFETs. The device fabrication of bottom-gate top-contact DPA single-crystal transistors followed the conventional procedures: (1) the heavily doped, $\mathrm{n}$-type $\mathrm{Si}$ wafer containing a 300 -nm-thick $\mathrm{SiO}_{2}$ layer was used as substrate, which was successively cleaned with pure water, hot acetone, pure ethanol and oxygen plasma (about $15 \mathrm{~s}$ );

(2) treatment of $\mathrm{Si} / \mathrm{SiO}_{2}$ wafer with OTS was then carried out by conventional vapour deposition method at a vacuum chamber $(0.1 \mathrm{~Pa})$ at $120^{\circ} \mathrm{C}$ for $2.5 \mathrm{~h} ;(3)$ single crystals of DPA were in-situ grown on $\mathrm{OTS} / \mathrm{SiO}_{2} / \mathrm{Si}$ substrate by physical vapour transport in a horizontal furnace under $5 \mathrm{~Pa}$, with DPA powder in the high temperature zone $\left(160^{\circ} \mathrm{C}\right)$ and the substrate at low temperature zone $\left(50-60^{\circ} \mathrm{C}\right)$ for OFET fabrication; (4) an 'organic ribbon mask' technique ${ }^{29}$ was used to construct the devices with two perpendicular conducting channels based on an individual single crystal for anisotropic transport investigation. Under the assistance of micromanipulator 6150 probe station with a high-resolution microscope (magnification at $\times 40-200$ ), micrometre or sub-micrometre organic ribbon prepared in advance on other substrates was peeled off and put directly on a pre-deposited DPA crystal with its direction just along the $b$ or $c$ axis, and then another organic ribbon was laminated over the previous one by a $90^{\circ}$, that is, $c$ or $b$ axes; (5) the substrate was transferred to a vacuum chamber and $50 \mathrm{~nm}$ gold was deposited on the masked single crystals; (6) the devices were finished by peeling off the ribbons. Organic field-effect transistor characteristics were obtained at room temperature in air on a Keithley 4200 SCS and Micromanipulator 6150 probe station and the mobility was extracted from the saturation region by using the equation of $I_{\mathrm{DS}}=(W / 2 L) C_{i} \mu\left(V_{\mathrm{G}}-V_{\mathrm{T}}\right)^{2}$.

Fabrication and measurement of DPA OLEDs. OLED devices were fabricated on ITO-coated glass substrate in a chamber with a pressure of $1 \times 10^{-4} \mathrm{~Pa}$. Electroluminescence spectra characteristics of OLED devices were tested and analysed in air. J-V-L characteristics were measured using a computer-controlled Keithley 2400 Sourcemeter. The EL spectra were measured with a spectrascan PR650 photometer.

Fabrication of integrated OFET-driving OLED arrays. To construct the OFETdriving OLED arrays (AMOLED), we first fabricate OFETs based on ITO gate electrodes, which are patterned by photolithography (Suss MA6/BA6) and an etching process. Double layers of ALD-deposited $\mathrm{HfO}_{2}(30 \mathrm{~nm})$ and spin-coated polyamide acid (PAA) $(300 \mathrm{~nm}$ ) are used as gate dielectric with a capacitance of $20 \mathrm{nF} \mathrm{cm}^{-2}$ calculated through Keithley $590 \mathrm{CV}$ analyser. Then, $50 \mathrm{~nm}$ DPA active layer was vacuum evaporated with $40 \mathrm{~nm}$ vacuum-deposited $\mathrm{Au}$ on top used as the source/drain electrodes. For OLED arrays, 40 or $20 \mathrm{~nm}$ semitransparent Au layer contacted the drain electrode of OFETs is vacuum-deposited on the PAA layer as the anode of the OLEDs. Subsequently, NPB/DPA/ TMPYPB/ LiF/ Al were vacuum thermally evaporated on the ITO anode in sequence. Finally, $\mathrm{Cu}$ was thermally evaporated on top for the data bus lines. The channel length and width of OFETs are 50 and $4,000 \mu \mathrm{m}$, respectively. The light-emitting area of OLED arrays is $400 \times 400 \mu \mathrm{m}^{2}$. AMOLEDs characteristics are tested and analysed in air.

\section{References}

1. Birks, J. B. Photophysics of Aromatic Molecules (Wiley, 1970).

2. Turro, N. J., Ramamurthy, V. \& Scaiano, J. C. Modern Molecular Photochemistry of Organic Molecules (University Science Books, 2010).

3. $\mathrm{Hu}, \mathrm{W}$. Organic Optoelectronics (Wiley-VCH, 2012).

4. Tang, C. W. \& VanSlyke, S. A. Organic electroluminescent diodes. Appl. Phys. Lett. 51, 913-915 (1987).

5. Burroughes, J. H. et al. Light-emitting diodes based on conjugated polymers. Nature 347, 539-541 (1990).

6. Ma, D. et al. A dinuclear aluminum 8-hydroxyquinoline complex with high electron mobility for organic light-emitting diodes. Appl. Phys. Lett. 82, 1296-1298 (2003).

7. Geens, W. et al. Dependence of field-effect hole mobility of PPV-based polymer films on the spin-casting solvent. Org. Electron. 3, 105-110 (2002).

8. Bao, Z. \& Locklin, J. Organic Field-Effect Transistors (CRC Press Taylor and Francis Group, 2007).

9. Klauk, H. Organic Electronics: Materials, Manufacturing and Applications (Wiley-VCH, 2007).

10. Sundar, V. C. et al. Elastomeric transistor stamps: reversible probing of charge transport in organic crystals. Science 303, 1644-1646 (2004).

11. Jurchescu, O. D., Baas, J. \& Palstra, T. T. M. Effect of impurities on the mobility of single crystal pentacene. Appl. Phys. Lett. 84, 3061-3063 (2004).

12. Hepp, A. et al. Light-emitting field-effect transistor based on a tetracene thin film. Phys. Rev. Lett. 91, 157406 (2003).

13. Sirringhaus, H. Device physics of solution-processed organic field-effect transistors. Adv. Mater. 17, 2411-2425 (2005).

14. Zaumseil, J., Donley, C. L., Kim, J.-S., Friend, R. H. \& Sirringhaus, H. Efficient top-gate, ambipolar, light-emitting field-effect transistors based on a green-light-emitting polyfluorene. Adv. Mater. 18, 2708-2712 (2006).

15. Samuel, I. D. W. \& Turnbull, G. A. Organic semiconductor lasers. Chem. Rev. 107, 1272-1295 (2007)

16. Namdas, E. B. et al. Low thresholds in polymer lasers on conductive substrates by distributed feedback nanoimprinting: progress toward electrically pumped plastic lasers. Adv. Mater. 21, 799-802 (2009).

17. Cornil, J. et al. Influence of interchain interactions on the absorption and luminescence of conjugated oligomers and polymers: a quantum-chemical characterization. J. Am. Chem. Soc. 120, 1289-1299 (1998).

18. Cao, X. et al. Self-assembly of twisted tetrachloroperylenediimide chromophores into two dimensional brick-stone aggregates: exciton dynamics and photoconductivity. Chem. Commun. 48, 6402-6404 (2012).

19. Laudise, R. A., Kloc, C., Simpkins, P. G. \& Siegrist, T. Physical vapor growth of organic semiconductors. J. Cryst. Growth. 187, 449-454 (1998).

20. Varughese, S. Non-covalent routes to tune the optical properties of molecular materials. J. Mater. Chem. C 2, 3499-3516 (2014).

21. Xu, Z. et al. Water-miscible organic J-aggregate nanoparticles as efficient two-photon fluorescent nano-probes for bio-imaging. J. Mater. Chem. 22, 17737-17743 (2012).

22. Yoon, S. J. et al. Multistimuli two-color luminescence switching via different slip-stacking of highly fluorescent molecular sheets. J. Am. Chem. Soc. 132, 13675-13683 (2010).

23. Spano, F. C. The spectral signatures of frenkel polarons in $\mathrm{H}$ - and J-aggregates. Acc. Chem. Res. 43, 429-439 (2009).

24. Smith, M. B. \& Michl, J. Singlet fission. Chem. Rev. 110, 6891-6936 (2010).

25. Xie, Z., Yang, B., Liu, L. \& Ma, Y. J-type dipole stacking and strong $\pi-\pi$ interactions in the crystals of distyrylbenzene derivatives: the crystal structures, high luminescence properties and prediction of high mobility. J. Mol. Eng. Mater. 01, 1340002 (2013).

26. Dong, H., Wang, C. \& Hu, W. High performance organic semiconductors for field-effect transistors. Chem. Commun. 46, 5211-5222 (2010).

27. Wang, C. et al. Semiconducting $\pi$-conjugated systems in field-effect transistors: a material odyssey of organic electronics. Chem. Rev. 112, 2208-2267 (2012).

28. Jiang, L. et al. Organic single-crystalline ribbons of a rigid " $H$ "-type anthracene derivative and high-performance, short-channel field-effect transistors of individual micro/nanometer-sized ribbons fabricated by an "organic ribbon mask" technique. Adv. Mater. 20, 2735-2740 (2008).

29. Lv, A. et al. High performance n-type single crystalline transistors of naphthalene bis(di carboximide) and their anisotropic transport in crystals. Chem. Commun. 48, 5154-5156 (2012).

30. Shi, J. \& Tang, C. W. Anthracene derivatives for stable blue-emitting organic electroluminescence devices. Appl. Phys. Lett. 80, 3201-3203 (2002).

31. Sirringhaus, H., Tessler, N. \& Friend, R. H. Integrated optoelectronic devices based on conjugated polymers. Science 280, 1741-1744 (1998).

32. Brown, A. R., Pomp, A., Hart, C. M. \& de Leeuw, D. M. Logic gates made from polymer transistors and their use in ring oscillators. Science 270, 972-974 (1995). 
33. Li, L. Q. et al. An ultra closely pi-stacked organic semiconductor for high performance field-effect transistors. Adv. Mater. 19, 2613-2617 (2007).

34. Giri, G. et al. Tuning charge transport in solution-sheared organic semiconductors using lattice strain. Nature 480, 504-508 (2011).

35. McRae, E. G. \& Kasha, M. Enhancement of phosphorescence ability upon aggregation of dye molecules. J. Chem. Phys. 28, 721-722 (1958).

36. Kasha, M. Energy transfer mechanisms and the molecular exciton model for molecular aggregates. J. Radiat. Res. 20, 55-71 (1963).

37. Zhai, D., Xu, W., Zhang, L. \& Chang, Y.-T. The role of "disaggregation" in optical probe development. Chem. Soc. Rev. 43, 2402-2411 (2014).

38. Lakowicz, J. R. Principles of Fluorescence Spectroscopy (Springer, 2006).

39. Cornil, J., Beljonne, D., Calbert, J. P. \& Bredas, J. L. Interchain interactions in organic pi-conjugated materials: impact on electronic structure, optical response, and charge transport. Adv. Mater. 13, 1053-1067 (2001).

\section{Acknowledgements}

We acknowledge Weigang Zhu in ICCAS for the discussion of the aggregation states. We acknowledge National Natural Science Foundation of China (51222306, 61201105, 91027043, 91222203, 91233205, 91433115), the China-Denmark Co-project (60911130231), TRR61 (NSFC-DFG Transregio Project), the Ministry of Science and Technology of China (2011CB808405, 2013CB933403, 2013CB933500), the Strategic Priority Research Program of the Chinese Academy of Sciences (XDB12030300) and Beijing Local College Innovation Team Improve Plan (IDHT20140512). A.J.H. acknowledges the financial support from the Heeger Beijing R\&D Center (Beijing, China), the 111 project (B14009) and the International Science \& Technology Cooperation Program of China (No. 2014DFA52820).

\section{Author contributions}

H.D., H.W. and A.J.H. designed the experiments. J.L. synthesized the compound. J.L., H.Z., L.J. performed the single crystal OFET experiments. J.L., L.M. and Y.W. helped to make the OLED devices. L.F.J., H.D. and J.Y. designed and performed the OFETOLED experiment. All the authors participated in the result discussion. H.D., W.H. and A.J.H. wrote the manuscript.

\section{Additional information}

Supplementary Information accompanies this paper at http://www.nature.com/ naturecommunications

Competing financial interests: The authors declare no competing financial interests.

Reprints and permission information is available online at http://npg.nature.com/ reprintsandpermissions/

How to cite this article: Liu, J. et al. High mobility emissive organic semiconductor. Nat. Commun. 6:10032 doi: 10.1038/ncomms10032 (2015).

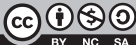

This work is licensed under a Creative Commons AttributionNonCommercial-ShareAlike 4.0 International License. The images or other third party material in this article are included in the article's Creative Commons license, unless indicated otherwise in the credit line; if the material is not included under the Creative Commons license, users will need to obtain permission from the license holder to reproduce the material. To view a copy of this license, visit http:// creativecommons.org/licenses/by-nc-sa/4.0/ 